\title{
Guest editors' foreword: special section on local pattern mining in graph-structured data
}

\author{
Tanya Berger-Wolf • Tamás Horváth
}

Published online: 28 January 2014

(C) Springer International Publishing Switzerland 2014

Mining graph-structured data has developed, over the last decade, into a separate research field of Data Mining and Knowledge Discovery. Examples of real-world graph-structured data include the Web graph, social, biological, telecommunication, utility, road and transportation networks, as well as relational data. The digital universe is producing an avalanche of these and many other occurrences of graph-structured data. Accordingly, there is an increasing demand for effective automated processing of massive graph-structured data. In this field of research, there is a distinguished interest in mining local patterns, i.e., patterns that are characteristic only for a subset of the input data, but still "interesting" according to some user specified criteria. Such local patterns can be utilized in various ways in diverse data processing tasks. For example, frequent patterns, the most common type of local patterns, proved to be useful ingredients for predictive and descriptive data analysis, for information retrieval, of for understanding the connection between the structure and the function.

We are pleased to present this special section on Local Pattern Mining in GraphStructured Data, with a particular focus on the formal aspects of this field. Out of the eight submissions we received in response to the call for papers, two papers have been selected for this special section.

The paper Mining Closed Patterns in Relational, Graph and Network Data by G. Garriga, R. Khardon, and L. De Raedt extends the notion of closed frequent patterns from itemsets to graphs and other relational structures. The authors investigate different problem settings arising from the combinations of the type of the input data and the choice of the pattern

T. Berger-Wolf

Department of Computer Science, University of Illinois at Chicago, Chicago, IL, USA

e-mail: tanyabw@uic.edu

T. Horváth

Department of Computer Science, University of Bonn, Bonn, Germany

T. Horváth $(\triangle)$

Fraunhofer IAIS, Sankt Augustin, Germany

e-mail: tamas.horvath@iais.fraunhofer.de 
matching operator. The input may consist of a set of disjoint small graphs (relational structures) or a single large graph (relational structure), while the matching operator is either subgraph isomorphism (substructure isomorphism) or graph (relational) homomorphism. For the four settings obtained from these combinations, they systematically investigate the uniqueness of the closures of patterns, as well as the adaptability of a state-of-the-art closed frequent itemset mining algorithm, the LCM algorithm, to these more general settings. One of the remarkable features of the LCM algorithm is that, in contrast to many related algorithms, it does not have to store the closed frequent itemsets already generated and, thus, can be efficiently generalized.

The paper A Polynomial-time Maximum Common Subgraph Algorithm for Outerplanar Graphs and its Application to Chemoinformatics by L.Schietgat, J. Ramon, and M. Bruynooghe is concerned with computing maximum common subgraphs of two outerplanar graphs. This problem is motivated practically by the observation that the molecular graphs of most of the known pharmacological molecules are outerplanar. Although this graph class has a number of desirable algorithmic properties, subgraph isomorphism and, thus, the maximum common subgraph problem as well, remains NP-complete for outerplanar graphs. To overcome this problem, the authors consider a constrained subgraph isomorphism operator which maps biconnected blocks to biconnected blocks and bridges to bridges. They show that the maximum common subgraph problem between outerplanar graphs can be solved in polynomial time for this particular operator. Besides this theoretical result, they experimentally demonstrate the utility of a distance metric based on maximum common subgraphs on quantitative structure activity relationship problems.

We would like to thank all authors for submitting their papers and the referees for their thorough work. We are particularly thankful to Martin Charles Golumbic for providing the opportunity to prepare this special section.

Tanya Berger-Wolf

Tamás Horváth 